\title{
Epistemología reciprocitaria. Aportes para un diálogo entre la antropología social y la investigación acción participativa
}

\author{
Reciprocal epistemology. Contributions for a Dialogue between Social Anthropology and Participatory Action \\ Research.
}

Jorge Luis Cladera

DOI: https://doi.org/10.24215/23468971e065

Universidad de Buenos Aires, Argentina

chorchcladera@gmail.com

Recepción: 07 Marzo 2019

Aprobación: 07 Noviembre 2019

\section{ResUMen:}

A partir de un esfuerzo por aproximar el programa epistemológico de la antropología social y el de la IAP, se observan las semejanzas y las diferencias que ambas establecen en torno a la categoría de participación. A continuación se realiza un ejercicio de extrañamiento etnográfico sobre dos experiencias de IAP realizadas con comunidades indígenas de Jujuy en las que el autor ha participado, lo que permite poner en evidencia dos roles diferentes de mediación social que resultan necesarios para procesos de IAP. Se propone que uno de esos roles, el de agente de estimulación, facilita accesos al campo social que permiten la integración del/ la investigador/a en instancias denominadas epitallerísticas y metatallerísticas. Se propone una manera alternativa de abordar el contrato epistemológico de la IAP que recupera el concepto de participación de la tradición etnográfica, permitiendo así un vínculo de reciprocidad epistemológica como alternativa a la coautoría en tanto producto de la IAP, por el cual se obtienen dos productos y no uno solo: siendo el producto innovador para los sectores subalternos, el enfrentamiento colectivo de las incompletitudes de su vida social.

Palabras clave: Etnografía, Investigación Acción, Participación, Promoción Social Rural.

\begin{abstract}
:
Through an effort to approach the epistemic program of social anthropology and that of Participatory Action Research (PAR), we observe the similarities and differences that both establish concerning the category of participation. An ethnographic estrangement exercise is carried out on two PAR experiences with indigenous communities of Jujuy (NW Argentina) in which the author has participated, thus highlighting two different mediation roles that occur in PAR. It is proposed that one of these roles, namely 'stimulation agent', may serve to the social researcher as a legitimate opening key to a given social field, by integrating him/her in instances that we name here as 'epi' and 'meta' workshop. Consequently, we propose an alternative way of considering the PAR epistemic contract, by recovering the concept of participation from ethnography, thus allowing an epistemological reciprocity bond between researcher and subaltern social subjects, by means of which two products, and not just one, may be obtained from the PAR experience: one of them being the scientific product - the paper -, which serves to the researcher; and the other one being a collective emancipation experience, which serves subaltern social subjects to ease certain aspects of their everyday livelihoods.
\end{abstract}

KEYWORDS: Ethnography, Research Action, Participation, Social Promotion.

\section{INTRODUCCIÓN}

La reflexión académica en Latinoamérica abunda en esfuerzos por dar entidad a una investigación social con vocación emancipadora. Una multiplicidad de experiencias de trabajo concretas con sectores populares ha brindado los insumos para repensar la relación entre participación, transformación social y quehacer científico, desde orígenes tan diversos como la comunicación social, la psicología comunitaria, la educación popular o la sociología. Distintos esfuerzos se han hecho por avanzar en el logro de síntesis disciplinares que le

NotAS DE AUTOR 
brinden cobijo y legitimidad conceptual a estas experiencias, bajo el marco de la llamada Investigación Acción Participativa -IAP, a partir de aquí-.

La antropología social se suma a estos debates no sin antes haber tenido que purgar ciertos resquemores conceptuales de su propio devenir histórico, tales como las sospechas de funcionalismo y colaboracionismo de la antropología para el desarrollo, o los recaudos para participar del cambio social dirigido, debido al etnocentrismo que un programa con este objetivo suele encubrir (Isla y Colmegna, 2005; Viola Recasens, 2000). Superando estos fantasmas disciplinares, recientemente se ha revitalizado el encuentro entre investigar y transformar la realidad social, bajo los conceptos de etnografía colaborativa (Arribas Lozano, 2015; Carenzo y Fernández Álvarez, 2014; Fernández Álvarez y Carenzo, 2014; Rappaport, 2007), y de antropología activista (Leyva Solano, 2010; Sorroche y O'Hare en prensa).

En este artículo me interesa hacer un aporte a estos esfuerzos contemporáneos por aproximar a la antropología social con otras experiencias latinoamericanas de investigación para la transformación social. Con este fin, presentaré algunas reflexiones conceptuales emanadas de mi trabajo como técnico en promoción social rural entre los años 2007 y 2016 con comunidades indígenas de la provincia de Jujuy. En múltiples oportunidades durante este período de tiempo, me vi en la necesidad de emplear herramientas, metodologías o abordajes inspirados en la IAP, con los que me fui familiarizando desde la práctica, ya que mi disciplina de proveniencia es la antropología social. La IAP es una categoría polisémica, como aclaran sus propias/os teóricas/os. La interpretación que haré de la categoría a lo largo del artículo se basa en las conceptualizaciones de Maritza Montero (2006) y de M. Teresa Sirvent y Luis Rigal (2014), por haber sido las lecturas que más influyeron en los esfuerzos por reinventar la práctica de intervención de determinados organismos de desarrollo rural en Argentina, sobre todo el INTA (ver por ejemplo Boletín del IPAF s/f; Regazzoni, De Pascuale, Martinez y Virgili, 2011).

A los fines de ordenar el proceso lógico del artículo, comenzaré por hacer una revisión de las significaciones que guarda, tanto para la IAP como para la observación participante etnográfica, una categoría que ambos abordajes metodológicos comparten, pero con sentidos, como ilustraré, opuestos: la participación. Luego presentaré dos experiencias, enmarcadas metodológica y conceptualmente en la IAP, en las que me tocó participar. A continuación, un ejercicio de extrañamiento etnográfico sobre estas experiencias me permitirá poner de relieve algunos desafíos que, en los programas de IAP tal como están pensados hoy, no están resueltos y generan debilidades heurísticas. En mis ejemplos se observará que, en los hechos concretos, estos desafíos son sorteados por medio de determinados dispositivos no expresados explícitamente por el programa metodológico, a los que denomino instancias epitallerísticas y metatallerísticas, y agentes de estimulación, que en los hechos implican la incorporación de herramientas etnográficas complementarias a las propuestas IAP.

De la mano de estos insumos de análisis, podré finalmente esbozar los lineamientos conceptuales que fundamentan una propuesta original, a la que denomino epistemología reciprocitaria. Esta propuesta se diferencia de las modalidades disponibles de IAP, en que asume que la experiencia de encuentro entre investigadores/as y sectores sociales subalternos puede generar simultáneamente dos subproductos que respondan a dos expectativas distintas puestas en juego en la interacción: un producto teórico-académico por parte del cuerpo investigador, y una transformación material concreta por parte de los sujetos del campo social de estudio.

\section{EL CONCEPTO DE PARTICIPACIÓN SEGÚN LA IAP Y LA ANTROPOLOGÍA SOCIAL}

Existe, como he adelantado, una categoría metodológica que es fundamental tanto para las aproximaciones etnográficas como para la IAP: el concepto de participación. En una como en la otra, el recurso a este concepto expresa los esfuerzos por superar las limitaciones analíticas de los abordajes positivistas sobre lo humano. Es menester entonces repensar una huella epistemológica profunda que ha dejado la racionalidad iluminista en las ciencias sociales: me refiero a la unidad cognoscitiva de la ciencia, constituida por el 
individuo que se enfrenta, mediante sus dispositivos sensibles, al conocimiento del mundo previsualizado en consecuencia como aquello que está por fuera de sí mismo. Esta piedra fundacional de las ciencias modernas ha establecido la imagen arquetípica que prefigura el quehacer del científico: el ojo que, por medio del dispositivo tecnológico, analiza y comprende el mundo.

La concepción del conocer como emanación lógica de la información obtenida mediante la observación externa y objetiva del mundo ha marcado desde entonces a todas las ciencias, incluyendo a las sociales. Esto sugiere un vínculo implícito entre el investigador/a y la información obtenida en el campo, que se podría ilustrar con el gráfico N. ${ }^{\circ} 1$.

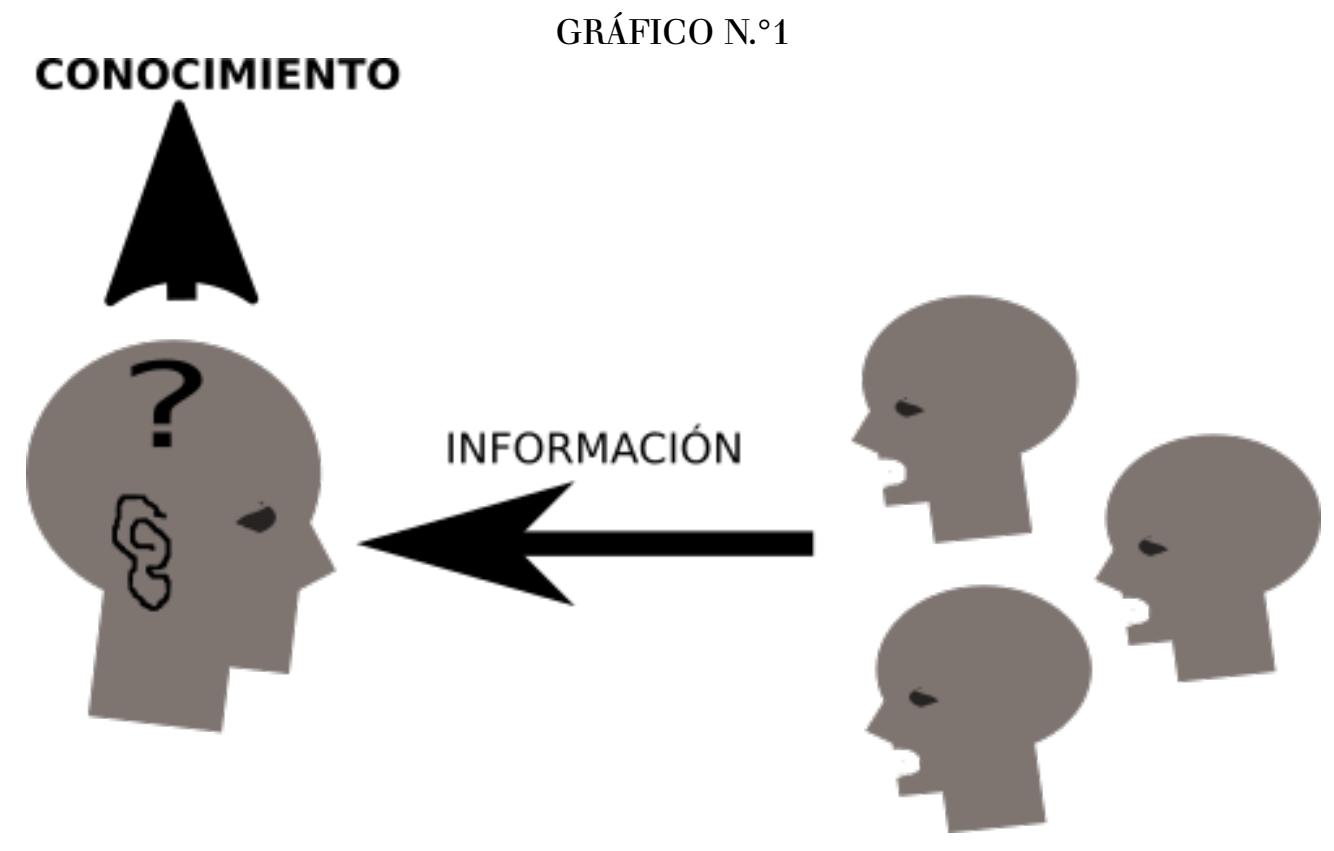

Siguiendo este razonamiento, la investigación constituiría un movimiento epistemológico unidireccional. El/la investigador/a se enfrenta a sus preguntas de investigación, y para contestarlas debe conocer a otros/ as sujetos/as y sus situaciones y contextos. Para ello iniciaría un movimiento en principio unidireccional, no interactivo, asocial, para el conocimiento acerca de lo social, es decir, acerca de interacciones recíprocas y bidireccionales: lo que provoca una paradoja entre el movimiento epistemológico y sus resultados.

A contramano de este modelo sintético, resulta inevitable que los/as cientistas sociales establezcamos con los campos sociales bajo estudio relaciones precisamente sociales, en las que por lo tanto los vínculos con los sujetos del campo deben ser recíprocos para construir conjuntamente determinada accesibilidad al campo y determinados criterios acerca de lo que es el conocimiento. En las aproximaciones de las ciencias sociales clásicas, la relación reciprocitaria del investigador y los sujetos de estudio no era un tema metodológico relevante, quedando en consecuencia librado al albedrío y a los procedimientos y pequeñas negociaciones locales que cada invesigador/a pudiera lograr, como se presenta en el gráfico N. ${ }^{\circ} 2$. 


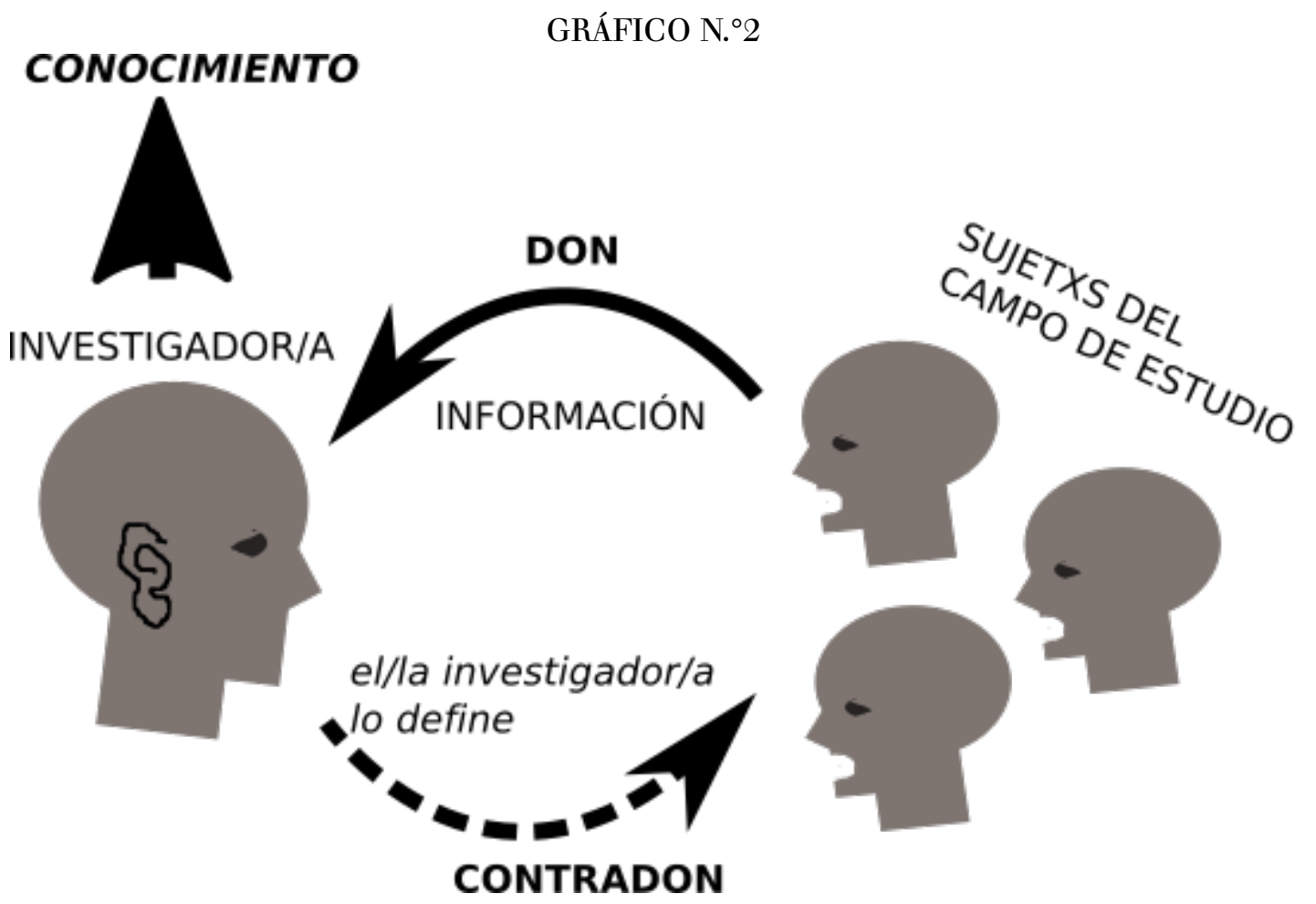

En el gráfico se manifiestan dos desafíos que enfrentan los estudios sociales en su interacción con sus campos de análisis social: el problema de la reciprocidad entre investigador/a y sujetos del campo de estudio; y el problema de la autoría sobre el conocimiento de lo social. Ambos problemas, aunque emparentados, son distintos, y han sido enfrentados de manera distinta por las dos trayectorias disciplinares que hoy nos involucran, empleando para ello una misma categoría metodológica con sentidos distintos: la de participación.

$\mathrm{Al}$ aproximarnos a cualquier "manual metodológico" de alguna de estas trayectorias disciplinares, lo primero que se advierte es la polisemia de estos conceptos, tanto para el caso de la Observación Participante etnográfica (Achilli, 2005; Boivin, Rosato y Arribas, 2007; Guber, 2004) como de la Investigación Acción Participativa (Montero, 2006; Rigal, 2008; Sirvent y Rigal, 2014). Tanto la una comola otra pueden significar muchas cosas dependiendo de la orientación, escuela o perfil del investigador/a que lo enuncie. Pero de manera más o menos sintética, podemos presentarlos como sigue.

La observación participante contiene dos categorías que hacen énfasis en dos acciones que en el trabajo de campo etnográfico están indisolublemente asociadas: observar y participar. Es decir, un movimiento de documentación o registro de hechos sociales (la observación); y un movimiento de integración del investigador en la recreación de dichos hechos sociales (la participación). Desde un enfoque interpretativista, el investigador adquiere plena comprensión de los fenómenos sociales bajo estudio al participar de las instancias en que estos ocurren. Los datos no están disponibles en el campo para ser recolectados por el investigador, sino que constituyen el producto emergente de interacciones sociales entre investigador/ a y sujetos del campo social, ya que la información registrada ha sufrido el proceso interpretativo del investigador: en la selección de lo visto y lo no visto, en el esfuerzo por poner en palabras y en otorgar coherencia a lo observado, en la atención puesta en las continuidades y en las inconsistencias verbales y/o comportamentales de los sujetos, y por último, en la atención puesta por el investigador en las modificaciones de los comportamientos y palabras de los sujetos frente a él mismo (la reflexividad). En todo caso, sin participar de las dinámicas sociales, el investigador no logra acceder a la comprensión de las tramas sociales que pretende conocer. Y sin tomar distancia analítica de esas mismas dinámicas -el extrañamiento- no puede abordarlas como objeto de reflexión conceptual. En este doble movimiento de aproximación a y distanciamiento de los fenómenos sociales que le interesan al investigador, cobra sentido la propuesta 
metodológica de la observación participante. Aunque hoy hay cada vez más reflexiones acerca de los modos de co-teorización etnográfica entre investigadoras/es y sujetos del campo social (ver Batallán y García, 1992; Fernández Álvarez y Carenzo, 2014; Arribas Lozano, 2015), como principio epistemológico, la observación participante requiere tanto de una fusión de horizontes de conocimiento como de una segregación de los mismos: ya que el/la antropólogo/a necesita tomar distancia conceptual de lo que estudia para poder hablar de ello, al menos en determinados momentos del trabajo.

La IAP, o simplemente Investigación Participativa (IP) en sus comienzos, constituye un esfuerzo de síntesis, originalmente llevado adelante entre otros por Fals Borda ([1998] 2015), con el fin de validar formas de investigación social apuntadas a consolidar la emancipación social de los sectores subalternos y oprimidos por medio del desarrollo de una conciencia social crítica. Para esto, la IAP establece la necesidad de la participación real de los sujetos en el proceso investigativo, entendida como la capacidad de los sujetos de incidir “...sobre todos los procesos de la vida institucional y sobre la naturaleza de sus decisiones" (Sirvent, 2004, p. 18). Para lograrlo, el trabajo del investigador asume muchas características que lo acercan al rol de coordinador de grupo en el marco de acciones de educación popular (Sirvent y Rigal, 2014).

La aproximación de la IAP hace el esfuerzo por dotar de carne a aquel principio original de la filosofía dialéctica: la idea de que el conocimiento es, precisamente, el producto de un diálogo. El proceso epistemológico que se procura dar podría ilustrarse mediante el Gráfico N.³.

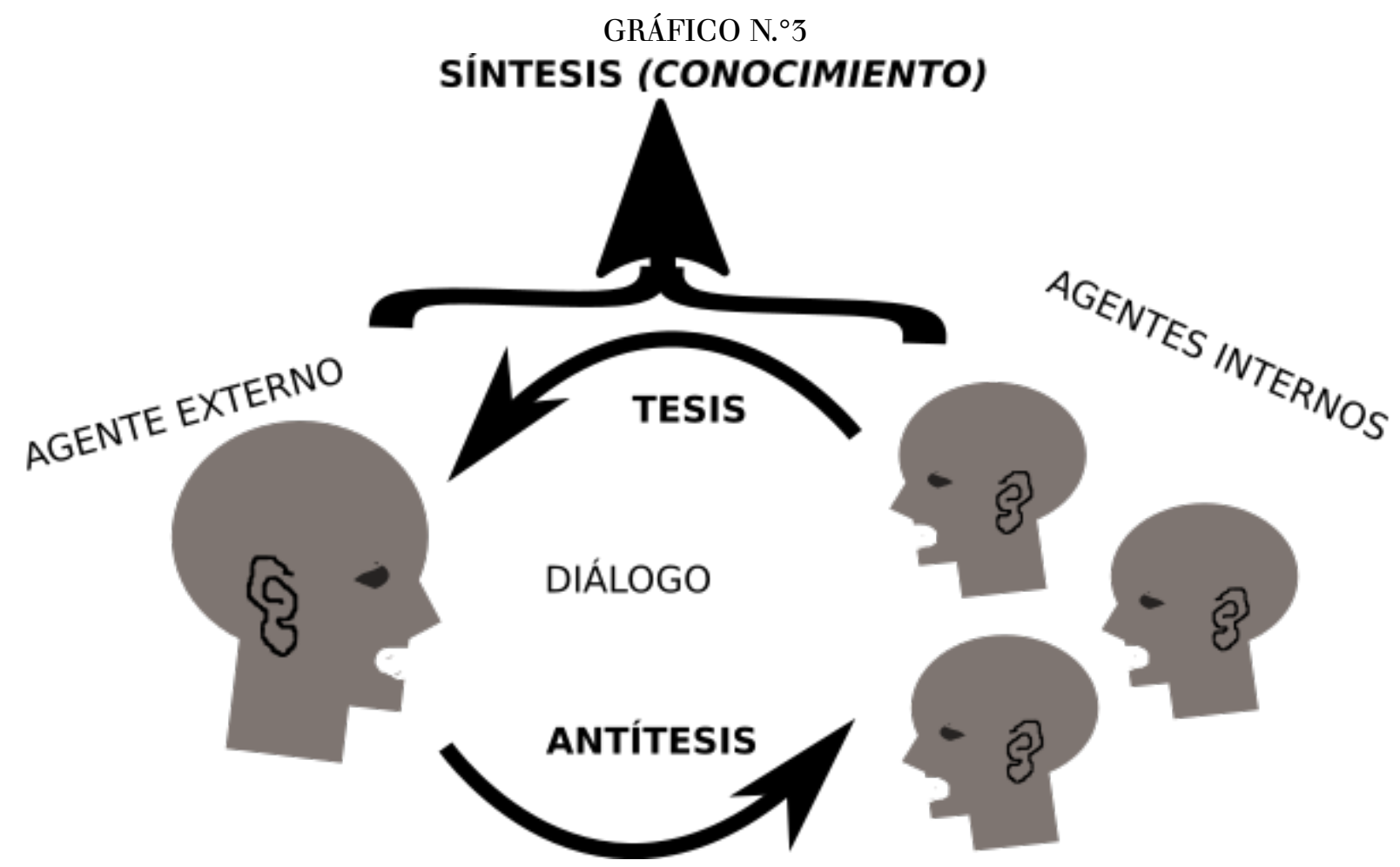

La IAP procura reconocer dos polos de una interacción productora del conocimiento social: los/las agentes externos al hecho social de estudio, y los/las agentes internos (Montero, 2006). Por lo tanto, el producto emergente, el conocimiento, es un hecho compartido y colectivo. Este conocimiento emergente es el resultado de una intención transformadora de lo social que motoriza la interacción entre agentes externos e internos. Es decir que, para la IAP, el objetivo constitutivo del estudio de la realidad es su transformación (Montero, 2006; Sirvent y Rigal, 2014).

Para lograrlo, el programa de la IAP establece que el involucramiento de los/las participantes de la experiencia bajo estudio debe darse en todas las instancias de la investigación: en la recopilación de la información, en el análisis de la información, en la reflexión superadora a partir de esa información analizada. 
La acción inicial del colectivo social produce experiencias que pueden ser abordadas para aprender de ellas, y mejorar de ese modo la acción futura. El ciclo epistemológico de la IAP consistiría entonces en el proceso: ACCIÓN - REFLEXIÓN SOBRE LA ACCIÓN - CONOCIMIENTO - NUEVA ACCIÓN (Montero, 2006).

Los esfuerzos por democratizar la información y por lograr una distribución horizontal y efectiva de la palabra por parte de las aproximaciones de IAP han dado como resultado dinámicas y técnicas (Vargas y Bustillos, 1984 y 1997) cuya eficacia las ha expandido más allá de las fronteras disciplinares, incluso hasta inspirar aproximaciones que ideológicamente se sitúan en las antípodas de la IAP, como por ejemplo, las dinámicas motivadoras empleadas en la concertación social.

En síntesis, lo que me interesa dejar en claro es que, en la procura por superar las limitaciones conceptuales de una aproximación positivista a lo social, dos trayectorias disciplinares distintas incorporan la categoría de participación. Pero el movimiento heurístico con que entienden la categoría es, en cada caso, exactamente inverso. Cuando el método etnográfico se refiere a la participación, alude al esfuerzo o movimiento del/ la investigador/a por involucrarse en los quehaceres cotidianos de "los otros", de los sujetos del campo social bajo análisis. Cuando la IAP menciona la idea de participación, hace referencia al esfuerzo del/la investigador/a por involucrar a los sujetos del campo social en el proceso de investigación.

Para lograr lo primero, el investigador debe sumergirse en cotidianidades ajenas, dejarse llevar por la corriente del campo social a conocer, sin saber de antemano, por lo tanto, qué contextos se volverán significativos en el transcurso de la propia experiencia. En cambio, para lograr lo segundo, el investigador debe establecer las condiciones contextuales adecuadas a la construcción colectiva del saber. El término más frecuentemente utilizado para designar estos espacios sociales con vocación emancipadora es taller. Incluso cuando las/os antropólogas/os incorporamos instancias para promover la co-investigación (Batallán y García, 1992, p. 86) o la co-teorización (Fernández Álvarez y Carenzo, 2014, p. 29) con los sujetos con los que trabajamos, el taller se constituye en el espacio adecuado para este trabajo horizontal y colectivo, para promover "instancias participativas de retroalimentación de la investigación con los grupos sociales investigados" (Sirvent, 2004, p. 4). Presentaré a continuación dos experiencias de IAP, y allí se pondrá en valor la articulación entre el momento tallerístico con situaciones extratallerísticas en las que considero que la observación participante ofrece un aporte a las herramientas participativas para la emancipación social.

\section{Dos ejemplos de Investigación Acción Participativa con comunidades indígenas EN JUJUY}

Desde el año 2007 me desempeñé como técnico de terreno con comunidades indígenas en la provincia de Jujuy, ${ }^{1}$ primero en relación a una agencia de extensión de INTA, y del 2011 al 2016, para la Subsecretaría de Agricultura Familiar del Ministerio de Agricultura, Ganadería y Pesca de la Nación. La categoría técnico de terreno no ha sido definida concretamente hasta donde tengo conocimiento: las tareas desempeñadas se relacionaban a aquellas que Freire ([1973] 2001) menciona como "comunicación rural" y Shugurensky (1989) como "promoción social": el eslabón más concreto de vinculación entre las comunidades locales y los programas institucionales de intervención. En mi trabajo como técnico de terreno, me ha tocado participar en múltiples situaciones que responden a los principios metodológicos de la IAP. Presentaré dos de ellas en particular, por ser aquellas en las que más explícitamente se organizó la metodología en base a estos principios.

Una de estas experiencias consistió en la denominada "Escuela Campesina de Ganadería del Zenta". Las Escuelas Campesinas constituyeron propuestas cuyo fin explícito consistía en implementar IAP por parte de equipos técnicos de agencias de extensión de INTA en el NOA. Tres equipos técnicos territoriales estuvieron a cargo de llevar adelante las propuestas: dos de ellos en el Valle Calchaquí salteño, y nuestro equipo, de reciente formación en aquel momento, en la Quebrada de Humahuaca. Este equipo estaba integrado por dos ingenieros/as agrónomos/as del PSA, ${ }^{2}$ y yo como becario de INTA, y habíamos comenzado a delimitar 
un socioterritorio de intervención técnica al que denominamos "el Zenta", por tratarse de comunidades localizadas en las laderas de las sierras subandinas homónimas, tradicionalmente criadoras de ganado mayor y menor a través de prácticas de trashumancia estacional entre distintas zonas ecológicas de dichas sierras.

La Escuela Campesina del Zenta tuvo vigencia durante dos años consecutivos, el primero de los cuales (2008) permitió diseñar y consolidar la propuesta que se llevó a cabo durante el segundo año. La primer Escuela del año 2008 constituía la mejor solución disponible a una demanda que había surgido fuertemente en los diagnósticos comunitarios: el abordaje del tema ganadero. Ni las familias que integraban las comunidades sabían expresar muy claramente qué esperaban de nuestro equipo técnico en torno al tema de "lo ganadero" -inspirados en algunas intuiciones o en experiencias de campañas antiaftosa, algunos integrantes se atrevían a aventurar tímidas sugerencias del estilo "traer toros de raza" o "conseguir vacunas"-, ni nosotros sabíamos muy bien cómo funcionaba la compleja vida agropastoril de las familias locales. Pero era una demanda que surgía recurrentemente. De manera que el aprender entre todas/os cómo era la vida ganadera en las comunidades indígenas, en el proceso de decidir qué cosas podíamos hacer juntos para mejorar la actividad, se mostraba en ese momento como una síntesis posible. Por lo tanto, el objetivo en el marco del cual se gestó la Escuela Ganadera en 2008 consistió en “conocer y comprender las estrategias de producción y de vida en la zona de trabajo, con el fin de revisar las estrategias de intervención actuales del INTA, y de propiciar nuevas metodologías y acciones de terreno en pos de un desarrollo territorial local." El objetivo no manifiesto -la currícula oculta- que nuestro equipo técnico quería desarrollar mediante la Escuela, era el de lograr una fusión de horizontes de conocimiento entre técnicas/os y comunidades indígenas, sobre la que desarrollar acciones localmente válidas para mejorar la cría de ganado, por medio de herramientas pedagógicas y comunicacionales acordes a nuestro público adulto no alfabetizado e históricamente oprimido.

Cabe aclarar que la ejecución de una propuesta de IAP colisionaba constantemente con los habitus y marcos conceptuales históricamente sedimentados en la propia institucionalidad de INTA. Nos vimos enfrentados a lograr el diálogo y promover una circulación horizontal de la palabra entre dos públicos sumamente heterogéneos. Por un lado, requeríamos desandar el habitus corporizado en los ingenieros agrónomos y veterinarios, para quienes era impensable arribar a un territorio sin un plan de acción y un cronograma de tareas preconcebidos. Por otro lado, nos enfrentábamos a colectivos sociales indígenas con una gran presencia de adultos no alfabetizados, y además reacios a brindar información sobre sus sistemas agropecuarios, debido a la aún viva memoria histórica del pago forzado de arriendos hasta principios de los 80 .

En consecuencia, fue necesario hacer concesiones para reducir susceptibilidades que nos permitieron la integración de equipos interdisciplinarios e intercomunitarios. Algo que no pudo ser completamente evitado, por ejemplo, fue cierto esquema de ordenamiento del conocimiento para la intervención que se encuentra muy arraigado en las formaciones profesionales de perfil agrotécnico: la organización en cuatro pilares de la producción denominados nutrición, manejo, sanidad y genética. La propia experiencia de las Escuelas demostró que estas cuatro categorías son permeables y están mediadas por otras formas de organizar la vida y el conocimiento en las cotidianidades indígenas con las que trabajábamos: pero habría sido imposible dar curso a la propuesta sin aceptar concesiones de este tipo.

La idea para la primer Escuela (la del año 2008) se presentó en las asambleas comunitarias mensuales de aquellas comunidades indígenas con las que habíamos iniciado procesos de autorización para el trabajo conjunto -Cianzo, Chorcán y Varas-. Fue la comunidad más poblada de las tres -Varas- la que se ofreció de anfitriona para realizar los tres talleres con que contó esta primer experiencia. Esto redujo la participación de las otras dos, ya que el traslado de integrantes de una comunidad a otra no era fácil. En el primer taller, participaron tres representantes por Cianzo -a los que pasamos buscando por el camino con la camioneta particular de uno de los miembros del equipo técnico- y ninguno por Chorcán. En el segundo, contamos con una entusiasta comitiva de cuatro integrantes de Chorcán -para lo que tuvimos que hacer el mismo esfuerzo- pero ya ninguno de Cianzo. Para llegar en horario a los talleres, no podíamos pasar por ambas 
comunidades para buscar a la gente, porque Cianzo y Chorcán no quedan sobre el mismo camino. El tercer taller lo realizamos ya exclusivamente con miembros de la comunidad de Varas.

Durante el primer taller realizamos una actividad de mapeado de los circuitos de traslado del ganado, por medio de tres maquetas que representaban el espacio de las sierras con sus dos laderas. El segundo taller trabajamos los síntomas de enfermedad animal mediante "maniquíes" ganaderos diseñados para la ocasión. La intención de estas dinámicas consistía en brindar las condiciones para que los conocimientos locales adquirieran sus propios canales de expresión, evitando la opresión metodológica que las herramientas escritas implicarían para nuestro público. Los resultados de estos talleres trascendieron las expectativas y evidenciaron dimensiones inesperadas vinculadas el afecto, la estética y el juego sobre las que ya reflexioné en otra oportunidad (Cladera, 2010).

En el tercer taller tuvimos que trabajar el tema de la aplicación de medicamentos (antiparasitarios y suplementos nutricionales). Este taller fue necesario para responder a la demanda insistente de las propias familias ganaderas, cuyos rebaños comenzaban a llegar en la fecha en que se realizó -el mes de diciembreextenuados por los largos traslados desde las tierras bajas de invernada.

A partir de los tres talleres del año 2008, nuestro equipo técnico pudo afilar mejor el lápiz para, al año siguiente (2009), ofrecer una propuesta más efectiva. Lo primero que definimos fue un equipo técnico predispuesto a dar curso a la propuesta - para lo cual fue fundamental la colaboración del entonces PSA-, un conjunto de comunidades destinatarias y una modalidad específica de participación, con la intención de garantizar, por un lado, la transmisión a nivel local de las experiencias y aprendizajes compartidos en la instancia IAP, y por otro, asegurar la continuidad en la participación que evitara interrupciones en el proceso pedagógico-investigativo. Habíamos observado que en el año 2008 la rotación de las/os participantes era tan grande que cada taller conversábamos con un público diferente. Nos dimos cuenta de que, si queríamos establecer instancias de construcción del conocimiento verdaderamente horizontales con nuestro público destinatario, necesitábamos en primer lugar contar con un colectivo interlocutor continuo, conocedor y predispuesto. No podíamos entrar de buenas a primeras proponiendo construir un conocimiento horizontal, con un público cuyas experiencias de integración a instancias institucionales -la escuela, el servicio militar, los sistemas de salud- estaban mediadas por una jerarquía de saberes completamente naturalizada, indiscutible e incluso deseada (la máxima aspiración para una madre pastora era que su hijo llegara a ser gendarme o policía). Por el contrario, necesitábamos presentar una propuesta que, tal como señalaba Freire (2006), partiera desde esa experiencia (partir de lo que es), para ir deconstruyéndola en el transcurso del proceso pedagógico-investigativo conjunto (para llegar a concebir lo que no es pero podría ser). Para eso, diseñamos y presentamos en las asambleas comunitarias una propuesta consistente en ofrecer una formación para dos promotores/as ganaderos/as por cada comunidad, destinada a ocho comunidades indígenas que compartían prácticas de territorialidad ganadera comparables: las comunidades de Cianzo, Aparzo, Palca de Aparzo, Chorcán y Varas (del departamento de Humahuaca, Jujuy); las comunidades de Caspalá y Santa Ana (del departamento de Valle Grande, Jujuy); y la comunidad de Volcán Higueras (del departamento de Iruya, Salta). Presentamos la idea en las ocho asambleas comunitarias, señalando que cada comunidad designara a sus propios/as promotores/as ganaderos. Podrían participar más integrantes de la comunidad, pero los/as designados/as estaban obligados a hacerlo, ya que pesaba sobre ellos/as una responsabilidad comunitaria; a cambio, la institución se hacía responsable de los costos y las gestiones logísticas para el traslado de las/os participantes. Estos/as promotores/as contarían con un diploma expedido por INTA y PSA/SAF, conforme habían cumplido con el proceso pedagógico.

En el primer encuentro, el equipo técnico propuso que cada taller se realizara en una comunidad diferente, cosa que fue aceptada por los/las participantes. En total se realizaron siete talleres que pasaron por siete de las ocho comunidades integrantes de la propuesta. Cada taller tuvo una duración de dos jornadas: la primer jornada centrada en actividades áulicas; la segunda centrada en visitas a campo de tropas de hacienda de un/ a vecino/a de la comunidad anfitriona. Esta modalidad de trabajo resultó fundamental para poder desplegar 
la fusión de horizontes de conocimiento que deseábamos: ya que, como habíamos comprobado en 2008, sólo en situaciones concretas dentro del corral y mediante la manipulación de los animales se presentaban las condiciones contextuales en las que adquirían verbalización y expresividad, y por lo tanto podían disputar poder, los saberes locales. Esta característica de los talleres exigía el pernocte en la propia comunidad anfitriona de todos los "escuelinos" (como se empezó a autodesignar el colectivo humano conformado por técnicos/as y promotores/as ganaderos/as participantes). Esto fue posible mediante el empleo de las instalaciones escolares de las escuelas albergue, de los salones comunitarios, e incluso de las casas particulares. Cada comunidad debía gestionar la comida y cocinar para proveer a los/as participantes durante las dos jornadas de trabajo.

El orden de los talleres se realizó de la siguiente manera: un primer taller (en la comunidad de Cianzo en junio/09) se centró en el diagnóstico territorial y en la historia de la ganadería en la región. El segundo y tercer talleres (en las comunidades de Varas y Chorcán, en agosto y septiembre/09) se enfocaron en la sanidad animal. El cuarto y quinto talleres (en las comunidades de Santa Ana y Caspalá: octubre y noviembre/09) se centraron en alimentación y forraje. Y por último, el sexto y séptimo talleres (en las comunidades de Aparzo y Volcán Higueras: en diciembre/09 y abril/10) se centraron en manejo reproductivo y genética.

En todo el transcurso del proceso pedagógico participamos en calidad de equipo técnico: cuatro ingenieros/as agrónomos/as, dos veterinarios/as, un ingeniero zootecnista y el autor de este artículo. Como promotores rurales por sus respectivas comunidades, participaron: un varón por Aparzo, uno por Palca de Aparzo, uno por Cianzo y uno por Caspalá; tres varones y una mujer por Santa Ana y la misma proporción por Varas; dos mujeres y dos varones por Chorcán; y dos varones por Volcán Higueras. Totalizando un equipo pedagógico-investigativo de 26 "escuelinos".

El otro ejercicio de IAP que sirve de insumo para las reflexiones de este artículo fue un proceso de Sistematización de Experiencias de la Agricultura Familiar. La sistematización de experiencias constituye una modalidad específica de Investigación Participativa (ver Verger Planells s/f), en tanto procura lograr una interpretación crítica de determinados procesos sociales, por medio de su historización y análisis realizados por parte de los propios actores involucrados en dichos procesos, con el fin de incidir en sus prácticas futuras (Jara s/f; Villavicencio, 2009). En el caso que estamos analizando, el objetivo de la propuesta general consistió, por un lado, en visibilizar y difundir las actividades territoriales que la Subsecretaría de Agricultura Familiar (SsAF) llevaba a cabo en localidades aisladas de todas las provincias. El segundo objetivo de la propuesta consistió en brindar los aportes metodológicos para que fueran los propios equipos técnicos y destinatarios territoriales los que pudieran desarrollar reflexiones críticas acerca de las experiencias de intervención social en las que ellos mismos estaban participando (Equipo de Sistematización DATyC, 2013).

Con este fin, el equipo nacional de comunicación popular al que se le designó la tarea se propuso realizar un libro que compendiara la sistematización de una experiencia de promoción social rural en cada provincia. Cada equipo provincial debió designar entonces, por un lado, qué experiencia social se sistematizaría; y por otro lado, quién asumiría la coordinación del equipo sistematizador. La delegación de Jujuy me encomendó la tarea de coordinar la sistematización, y designó como experiencia social a analizar una que se localizaba en las antípodas de mis experiencias de trabajo como técnico. Se trataba de un proyecto de construcción de viviendas -el proyecto "Yaguata Kavi" (Vivir bien, en idioma Avá) - de la Comunidad denominada APG (Asamblea del Pueblo Guaraní) El Bananal, localizada en las inmediaciones del Parque Nacional Calilegua, en el departamento de Yuto. Cabe aclarar que todas mis experiencias de trabajo como técnico eran, hasta entonces, en comunidades rurales andinas del pueblo kolla; nunca antes había trabajado con el pueblo avá guaraní. Este proyecto consistió en 48 ampliaciones de vivienda y 3 instalaciones de baños en casas particulares, las que se lograron realizar entre septiembre de 2010 y mayo de 2011, a pesar de dos desafíos bastante difíciles de sortear: el proyecto carecía de recursos para cubrir mano de obra, y carecía de personal técnico especializado en construcción. 
El primer requisito que debimos resolver consistió en identificar al equipo sistematizador. Siguiendo la sugerencia más valorada por los manuales de sistematización disponibles (FAO, 2004; Villavicencio, 2009), decidimos componer nuestro equipo junto a las técnicas territoriales y a la organización social adjudicataria de la experiencia bajo análisis. Completaron así nuestro equipo sistematizador ocho mujeres, dos de ellas técnicas de terreno (la técnica de la SAF, y una técnica de ENDEPA: Equipo Nacional de Pastoral Aborigen) y seis integrantes de la propia Comunidad de El Bananal, que eran quienes habían coordinado las tres cuadrillas de construcción en que se había organizado la comunidad para cumplir con los plazos de ejecución del proyecto. El eje de sistematización que nuestro equipo elaboró fue el siguiente: “¿Cuáles fueron los factores, experiencias y estrategias que permitieron la organización autogestionada del Proyecto Yaguata Kavi de la APG El Bananal?" (Ruiz et al., 2013, p. 226). Entendiendo por factores a "los condicionantes externos (actores sociales, personalidades, decisiones)"; por experiencias a "las circunstancias previas transitadas por la comunidad que arraigaron en la memoria práctica de la organización", y por estrategias, "las prácticas efectivas desplegadas o desarrolladas durante la ejecución del proyecto" (Ruiz et al., 2013, p. 226).

A medida que las reflexiones sobre la experiencia avanzaban, se hacía evidente que el indiscutible protagonismo que habían adquirido las mujeres de la comunidad en el desarrollo y ejecución de un proyecto de construcción de viviendas constituía el dato más significativo a comprender y poner en valor, y así fue como la sistematización del proyecto acabó por titularse "Mujeres albañiles" (Ruiz et al., 2013).

El proceso de construcción del corpus documental de nuestro equipo se llevó a cabo entre los meses de mayo y agosto de 2011, a razón de un taller de trabajo quincenal. Cada taller se realizó durante una jornada de trabajo, siempre en el salón comunitario de El Bananal, y en estas instancias se abordaron distintas técnicas dinamizadoras para reflexionar sobre la experiencia, tales como juegos de roles, líneas de tiempo, un taller FODA y un mapa parlante. Esta información fue complementada mediante la realización de un taller de Grupos Focales al cierre del año 2011, en el que participaron en plenario las 51 familias adjudicatarias de las obras de vivienda realizadas bajo el proyecto. Durante todo el año 2012, el equipo sistematizador continuó juntándose para redactar el documento final. El libro fue publicado y presentado en el año 2013 (Equipo DATyC, 2013).

Tanto la Escuela Campesina de Ganadería del Zenta, como la Sistematización de Experiencias de Yaguata Kavi en la comunidad APG El Bananal, contribuyeron sensiblemente a valorizar los espacios colectivos de construcción de conocimiento, en el plano personal del autor de este artículo y sin duda también en el plano de las subjetividades de los/as integrantes de los sectores populares que participaron de las experiencias. No obstante, existen significativas diferencias en términos de qué alcances pero también qué límites analíticos permiten las aproximaciones de IAP llevadas a cabo entre ambas experiencias, que dependen del contexto y rol de participación que en cada caso enmarcaban el quehacer del autor de este artículo.

\section{UN EJERCICIO DE EXTRAÑAMIENTO DE LAS EXPERIENCIAS DE IAP}

A continuación entonces propondré realizar un ejercicio de extrañamiento sobre las propias experiencias de IAP en que he tenido la oportunidad de involucrarme. Este ejercicio no tiene por intención desautorizar a la IAP como metodología, sino por el contrario, pretende visualizar las debilidades de su puesta en funcionamiento efectiva, precisamente para enfrentarlas y vigorizar así su programa heurístico.

Uno de los mayores desafíos metodológicos que observo en estos procesos analíticos se relaciona con la participación. Como se ilustró en los casos presentados, la idea de participación encierra dos significados subyacentes: asistencia, y circulación de la palabra. La distribución de capitales de determinado campo social (en un sentido bourdieano) es desigual. Las relativas posiciones de poder de cada sujeto dentro del campo social dependen de pequeñas alianzas, lealtades y opresiones microsociológicas que lo prefiguran. En consecuencia, la puesta en función de estas metodologías participativas tiene lugar en el marco de configuraciones de campo predeterminadas, que incidirán y afectarán a los alcances analíticos de la propuesta. 
Estos efectos de participación los observo en dos sentidos, a los que me permito llamar aquí, ascendente y descendente.

En relación a la dimensión ascendente de la participación, es necesario aclarar que esta será una preocupación sólo para algunas orientaciones epistémicas que inspiren a la propuesta de investigación participativa. La IAP gestada desde la educación popular, por ejemplo, cuyo objetivo explícito es la superación de las situaciones de opresión (Rigal, 2008; Sirvent y Rigal, 2014), no considera conducente la integración pedagógica de sectores hegemónicos y subalternos en un mismo espacio de trabajo ya que el principio ontológico de su propuesta es que sólo la liberación del oprimido liberará asimismo al opresor. En cambio, otras experiencias de investigación participativa que no parten de esta premisa conceptual, tales como por ejemplo las técnicas de concertación social y de modelización de acompañamiento (companion modelling) empleadas por agencias de cooperación europeas (Barreteau, Bousquet, Étienne, Souchère y d'Aquino, 2010; Slocum, Elliott, Heesterbeek y Lukensmeyer, 2006), se permiten establecer actividades de diseño participativo en que distintos sectores sociales integran un mismo espacio de diálogo o negociación.

En la aplicación de metodologías en contextos como estos últimos, uno de los grandes desafíos difíciles de superar es, precisamente, lograr la efectiva participación de los sectores sociales "ganadores" alrededor de la temática en cuestión. Si, por ejemplo, se definen talleres para la planificación territorial o para el manejo de una cuenca hídrica, resulta muy difícil lograr que participen de la propuesta los grandes propietarios de tierras o los mayores usuarios del agua. La razón es evidente por sí misma: siendo ganadores, nada hay que los estimule a participar de estos espacios de construcción colectiva de consensos, en los que sólo habrán de reducir su margen de beneficio relativo.

Entonces, las propuestas de investigación participativa, ya sea que partan desde una episteme emancipatoria o no, se enfrentan de antemano a una reducida o nula participación social ascendente.

Ahora bien, en sentido descendente también reconozco dificultades y debilidades de participación. Los sectores populares no son internamente homogéneos y esto repercute en las instancias de convocatoria, en las instancias de circulación de la información y en la asistencia continuada a lo largo del transcurso de la propuesta metodológica. El esfuerzo metodológico por la construcción del conocimiento colectivo pierde de foco una pregunta antropológica fundamental (Quirós, 2011): las trayectorias individuales que llevan a que las personas participen de la propuesta o no y que lo continúen haciendo en cada instancia de encuentro en el marco de la IAP. Particularmente ilustrativo respecto de esto último resulta lo señalado sobre la participación en la Escuela Campesina, el cual debió anclarse a pequeñas concesiones de determinados contradones concretos que estimularan el interés: sólo a modo de ejemplo, el éxito en la realización del tercer taller del año 2008 radicó en la aplicación de antiparasitarios y suplementos nutricionales, aunque no venía a colación del proceso de construcción colectiva de conocimiento que estábamos procurando desarrollar. No puedo dar ejemplos de las negociaciones que permitieron la sistematización de Yaguata Kavi, y esto debido a un hecho que explicaré un poco más adelante.

No todos/as los/as que participan lo hacen movidos/as por las mismas inquietudes. Pero, fundamentalmente, casi nadie lo hará movido por inquietudes epistémicas; es decir, por la intención de enriquecer el conocimiento. En el caso de la Escuela de Ganadería, debimos imponer en 2009 un criterio pedagógico en principio formal y riguroso inteligible a los intereses de nuestro público, para consolidar un público interlocutor fijo y constante que no pudimos obtener en el 2008.

Y aquí subyace la fragilidad ontológica del programa de la IAP: la heurística, el deseo de conocer, funciona como motorizador para los/as cientistas sociales por cuanto constituye para ellos/as (para nosotros/as) el interés utilitario puesto en juego en la interacción; pero no ocurre lo mismo para los sectores populares, que tienen urgencias materiales de otras características.

Cuando un espacio de IAP cuenta con una asistencia acentuada y avanza a buen paso, desde mi experiencia, se debe a motivos externos al propio proceso metodológico y, casi siempre, a pequeños factores de estímulo, lubricación y hasta coacción microsocial ejercidos por figuras clave de los sectores sociales, que lo hacen 
precisamente para garantizar la participación y llenar de presencia las propuestas de transformación social. En la Escuela Campesina, sólo a modo de ejemplo, he mencionado que el éxito del año 2009 dependió de asegurar los costos y las gestiones para el traslado de las/los integrantes desde sus comunidades de origen a la localidad de trabajo de cada ocasión; así como a la presión comunitaria para exigir la participación de aquellos integrantes que habían sido designados en representación de su comunidad. No he mencionado aquí la multiplicidad de charlas que fueron necesarias para desactivar conflictos entre parientes a fin de que estuvieran dispuestas/os a integrar conjuntamente instancias colectivas de trabajo.

Entonces, la vigencia de los talleres de IAP depende de la acción no visibilizada de determinados sujetos que actúan para mantener vivos los espacios colectivos, a los que denomino aquí agentes de estimulación. Me parece necesario establecer esta categoría específica, para distinguirla de otros roles de mediación social que en la bibliografía (Cowan Ros y Nussbaumer, 2011) aparecen como indistintos, tales como los "animadores sociales" o "coordinadores pedagógicos". En cambio, las/los agentes de estimulación son las/ los que mantienen vivo el espacio colectivo de IAP por medio de procedimientos que aquí denomino epitallerísticos (coordinar y colaborar en la logística, en los traslados de participantes, en la preparación de la comida, el té o el mate, en los recordatorios radiales y convocatorias verbales, en las aperturas y clausuras de los espacios colectivos, etc.) y metatallerísticos (observar, preguntar y conversar con los participantes acerca de las instancias de IAP, pero por fuera de dichas instancias; realizar las visitas casa por casa; suavizar enfrentamientos personales que interfieren en el proceso colectivo, etc.).

Como se ve, animador/a pedagógico/a y agente de estimulación pueden ser funciones ejercidas por la misma persona, o bien por personas diferentes. En las Escuelas Campesinas de Ganadería en el Zenta mi función fue, en determinados momentos de los talleres, la de animador pedagógico, pero siempre de agente de estimulación. En la sistematización del proyecto Yaguata Kavi, he ejercido de animador pedagógico pero no de agente de estimulación ya que no se trataba de mi territorio de pertinencia como técnico. De no haber sido yo técnico de terreno en otro territorio, probablemente no habría reparado en la sorprendente vigencia del entusiasmo de las seis coordinadoras de cuadrillas de trabajo coautoras de la sistematización o de la masividad de la convocatoria a los Grupos Focales. Podría autocelebrarme y asociar el éxito de la propuesta metodológica a fenómenos intrínsecos a la propia metodología. Pero, siendo técnico territorial, soy y era en ese momento consciente de que esa participación dependía de resortes metatallerísticos y epitallerísticos que descansaban sobre los esfuerzos del personal técnico de SAF y ENDEPA, sobre los cuidados cotidianos en el estímulo a lo colectivo y sobre pequeñas desactivaciones de conflictos sociales latentes para que no repercutieran sobre el proceso metodológico. La diferencia sustancial entre la Escuela Campesina de Ganadería y la Sistematización de Yaguata Kavi era que en la primera yo conocía y había sido uno de los tejedores de ese frágil y dinámico entramado social que permitía la vitalidad del colectivo. En la segunda, esos resortes me pasaban desapercibidos y me eran ajenos.

Las aprobaciones y desaprobaciones de las propuestas metodológicas, el éxito o fracaso en su implementación, la legitimidad del espacio colectivo, la satisfacción o insatisfacción que producen los animadores pedagógicos, no pueden evaluarse adecuadamente en las instancias áulicas o en las técnicas participativas, sino que se infieren a partir de las visitas casa por casa, de las conversaciones fuera del aula, de la masividad o debilidad de la asistencia, de la distribución de los participantes según categorías sociales (mujeres/varones; jóvenes/adultos/ ancianos), de la fluidez con que se desplaza la palabra, etc. Esta información puede ser recuperada por las/los agentes de estimulación, precisamente porque en su ejercicio de estimulación implementan estas herramientas epitallerísticas y/o metatallerísticas. En suma: los/ las agentes de estimulación practican una metodología equivalente a la de los/las etnógrafos/as: estudian los epifenómenos invisibles, para comprender mejor el fenómeno manifiesto (el taller grupal, la dinámica pedagógica, la asamblea comunitaria).

En todo caso, hay dos características fundamentales de la función de agente de estimulación que es necesario señalar: 
- los/las agentes de estimulación son los que detentan la autoridad sobre el espacio colectivo. Para que un/a animador/a pedagógico/a sea imbuido con dicha autoridad, obran pequeños rituales de transferencia de la autoridad: las "presentaciones". En la instancia de presentación ("hola, ellos son tales y cuales, vienen de tal lado para realizar tal cosa”), es el/la agente de estimulación quien está transfiriendo transitoriamente una parte de su propio capital simbólico a los animadores pedagógicos.

- Tanto la función de los/las agentes de estimulación como los dispositivos empleados por ellos/ as para mantener vigorosa la participación, suelen pasar inadvertidos, o por lo menos están subrepresentados, en los estudios y la bibliografía sobre mediación social, IAP y técnicas participativas en general. Cuando se publican los resultados de una experiencia de construcción participativa de conocimientos, los resultados, buenos o malos, son adjudicados a la propia metodología tallerística; mientras que los factores que permitieron mantener vivo el espacio colectivo hasta el final del proceso metodológico ni siquiera son mencionados (pueden consultarse ejemplos a lo largo de la bibliografía citada).

Por eso considero fundamental visibilizar que la mayor dificultad para llevar adelante procesos de IAP consiste en mantener el espacio social vivo, lleno. Para ello, la IAP no ofrece respuestas, sino que se vuelve dependiente de resortes que provienen de otras esferas sociales. La paradoja epistémica consecuente se puede poner en evidencia en los ejemplos arriba citados. Los espacios pedagógicos liberadores, antiopresores y constructores de lo colectivo, dependen para su continuidad y existencia de pequeñas instancias de lubricación y coacción microsocial ejercidas por sujetos comprometidos con esos espacios, que cuentan sin embargo con un capital social o cultural ligeramente desigual que les permite por eso mismo ejercer esa capacidad de insuflar vitalidad al espacio colectivo. La Escuela Campesina sólo logró éxito al incorporar convenciones que remitían a una jerarquía de saberes que el propio espacio pretendía deconstruir: docente/ alumno; entrega de diplomas; obligatoriedad de asistencia; orden temático agrotécnico, etc. En cuanto se diluye o se debilita la capacidad de lubricar la participación que tienen los agentes de estimulación, estas mismas propuestas de IAP rápidamente languidecen y sus aprendizajes colectivos caen en desuso.

Esto ocurre, aunque no nos guste, por la inevitable vinculación utilitaria que establecen los sectores populares (cualquier persona, en realidad) con los espacios colectivos. Comprender por qué ocurre esto es precisamente lo que se espera de nosotros/as en tanto científicos/as sociales: comprender el "sentido práctico" de la cotidianidad, como lo denominaba Bourdieu (2007), o la "dimensión práctica de la vida social” para Gramsci (Nun, 1989; Rigal, 2011).

\section{OTRA MANERA DE PENSAR EL CONTRATO EPISTEMOLÓGICO DE LA IAP}

Hemos visto que los estudios abordados desde la IAP se esfuerzan por superar la trampa iluminista del vínculo unidireccional, por medio de la construcción colectiva del conocimiento. El producto final procura ser el resultado de un trabajo colectivo y compartido y, por lo tanto, la coautoría y la coteorización constituyen formas posibles de establecer la reciprocidad en el vínculo entre academia y campo social de análisis.

Ahora bien, los elementos que hemos tratado de poner de manifiesto hasta aquí acerca de nuestras propias experiencias nos permiten entrever algunas reflexiones.

La primera es que la preocupación por la autoría se relaciona con el dilema constitutivo de la ciencia. Centrar el debate alrededor de la autoría de un documento científico, pone de manifiesto la incomodidad de uno de los polos de la interacción social: el polo académico. El otro polo de la interacción -el campo social de análisis- no está necesariamente motorizado por esta paradoja. Consecuentemente, el producto final en coautoría no constituye necesariamente la única o la mejor manera de compensar las expectativas del otro. Esto nos permite redireccionar el debate no tanto hacia el tema de la coautoría, sino más bien hacia el plano de la reciprocidad. 
De aquí nace nuestra propuesta de repensar otra aproximación posible al concepto de IAP, a la que denomino epistemología reciprocitaria. El/la investigador/a en campo y el universo social de análisis se aproximan mutuamente movilizados por intereses diferentes, cada uno de ellos respondiendo a sus propias condiciones de reproducción material de la vida. El/la investigador/a pone en juego inquietudes epistemológicas, por cuanto estas constituyen su interés utilitario en la interacción. Los/as sujetos sociales de sectores subalternos ponen en juego otras inquietudes utilitarias, motivados por sus propias vulnerabilidades sociales y por la urgencia de las incompletitudes de su propia vida. Menciono aquí la idea de incompletitudes, ya que otros tipos de categorías sociales para designar la carencia -necesidades básicas insatisfechas, hacinamiento, indigencia, inseguridad alimentaria, informalidad económica, analfabetismo, etc. - responden a marcos conceptuales que predefinen desde arriba y desde afuera aquellos aspectos que se consideran requisito para una vida íntegra. La idea de incompletitudes que estoy proponiendo, nos invita a remitirnos a la experiencia subjetiva y culturalmente localizada de lo que está irresuelto para alcanzar el buen vivir.

Podríamos retomar el concepto de participación entonces, no de la tradición disciplinar de la IAP, sino de la tradición etnográfica de la observación participante, lo que invierte el movimiento heurístico del término. Para proponer una epistemología reciprocitaria, en lugar de tomar la participación como el esfuerzo por involucrar a los/as oprimidos/as en el proceso metodológico de la investigación, podemos pensar la participación como el esfuerzo del/la investigador/a por involucrarse en las incompletitudes de la vida de los/las oprimidos/as. Y en el proceso, ir construyendo un conocimiento etnográfico acerca de las tramas significantes de dicho campo social. Rigal (2008) propone la IAP como una instancia liberadora que transforma tanto al pedagogo/investigador como a los sujetos del campo social de estudio. En una abordaje de un campo social sobre la premisa que sugiero, los/las sujetos del campo social de acción son transformados/ as en la identificación, verbalización y enfrentamiento de incompletitudes, en cuyo proceso de resolución adquiere reivindicación valorativa la condición social subalterna. Pero el/la investigador/a también sufre una transformación en su rol: ya que la condición de investigador/a constituye sólo una dimensión específica de una inserción multifacética al campo social de interacción (Citro, 1999).

Si las preocupaciones de las ciencias sociales, y de la antropología social en particular, pasan por comprender las condiciones de posibilidad para el saber práctico, para la construcción de lo cotidiano (Achilli, 2005; Bourdieu, 2007), entonces una forma válida de sumergirse en esos campos de lo cotidiano consiste en compartir y acompañar procesos para enfrentar incompletitudes. Esta oportunidad de acompañar la identificación, verbalización y planificación colectiva alrededor de las incompletitudes de la vida cotidiana local por un lado, y su articulación estratégica para ejercer un "espionaje inverso" a favor de los sectores subalternos frente a distintos organismos institucionales (Sorroche y O'Hare, en prensa), constituye una oportunidad de apertura de campos etnográficos muy difícil de obtener por otros medios, por cuanto el contradon del vínculo es más transparente para los/as integrantes de la comunidad local. Una oportunidad que permite al/la investigador/a ejercer la observación participante en un rol dinámico: asomarse a ventanas concretas en las que los campos de lo cotidiano se despliegan ante él, en acciones que las reproducen y las transforman. No sólo en su condición de animador pedagógico, sino especialmente en su rol de agente de estimulación del espacio social, por medio del cual las instancias de trabajo colectivo, como las asambleas y talleres comunitarios, adquieran vigencia y vitalidad.

De esta manera cobra sentido la redefinición del concepto de participación que estamos proponiendo para un abordaje alternativo de instancias de IAP. El contrato epistemológico entre investigador/a y campo social de estudio se puede repensar y explicitar entonces como motorizado por intereses distintos, pero que pueden ser complementarios en un proceso reciprocitario. El movimiento epistemológico que estamos proponiendo se podría ilustrar mediante el gráfico n. $^{\circ} 4$. 


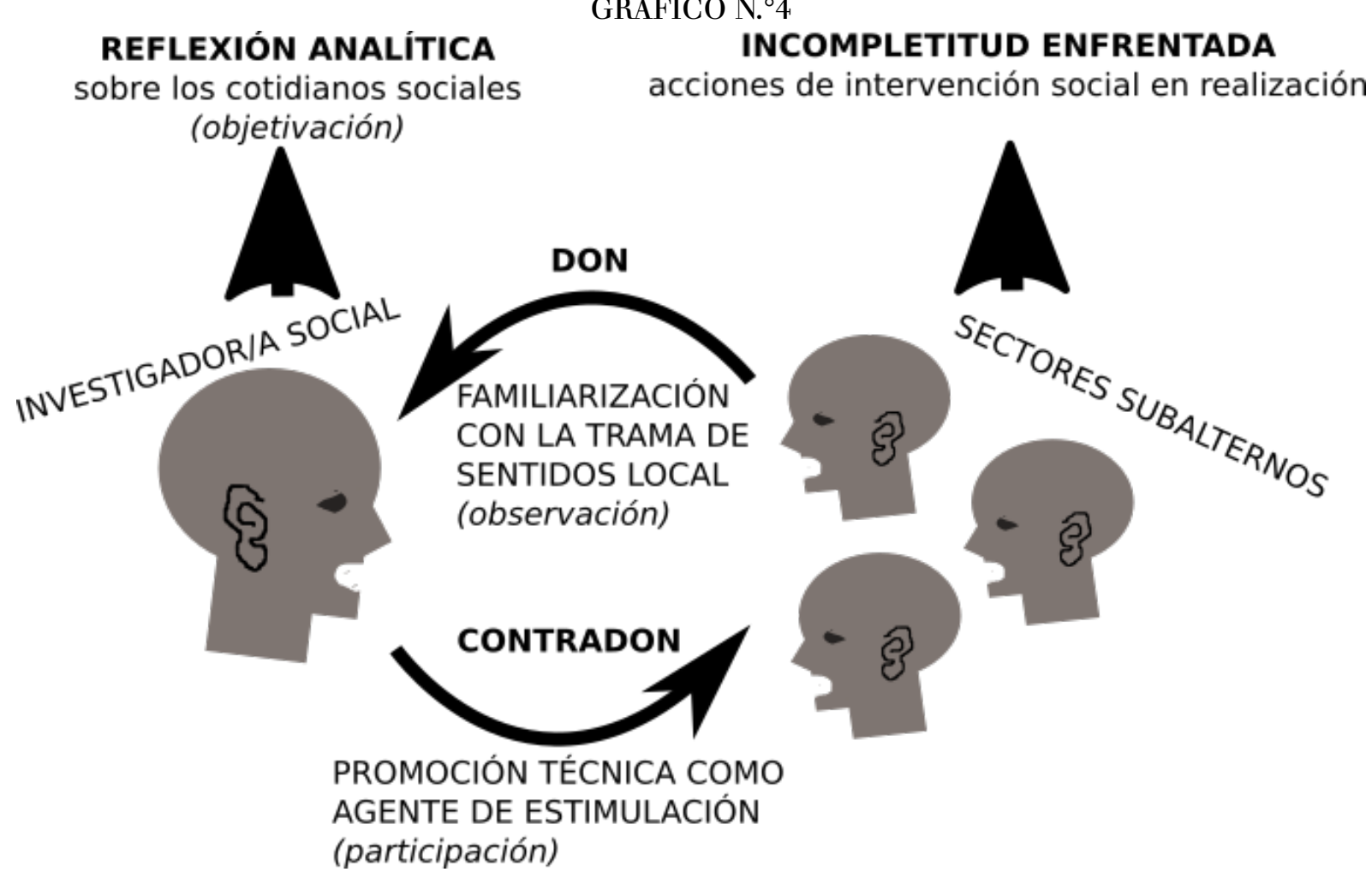

El desafío que se despliega para el quehacer del/la investigador/a social, en una aproximación epistemológica como la que estamos proponiendo, consiste en una apuesta redoblada sobre el esfuerzo que significa la metodología de la observación participante. El primero de estos esfuerzos consiste en el de familiarización y empatía con las tramas de sentido de las comunidades locales en la identificación de sus incompletitudes y del modo en que estas adquieren expresión. El segundo esfuerzo metodológico consiste en practicar una observación participante en el trabajo conjunto con aquellos/as otros/as sujetos con los que habremos de compartir las tareas en el proceso de resolución de dichas incompletitudes: el resto de un equipo técnico interdisciplinario. Ya en otra oportunidad Isla y Colmegna (2005) han observado que, dado el hecho de que la trama de sentidos local que da inteligibilidad a los problemas y demandas sociales es precisamente local, no existen formaciones disciplinares idóneas para acompañar su resolución y, por el contrario, los equipos técnicos van configurando dinámicas de trabajo en que las especificidades disciplinares se desdibujan en una realidad social que desafía constantemente el diseccionamiento hegemónico de las pertinencias profesionales.

Abordada desde estas dos dimensiones metodológicas, podríamos repensar la observación participante, inspirados en el movimiento dialéctico propuesto por la IAP: observar las incompletitudes -participar en su enfrentamiento- objetivar las configuraciones del sentido práctico y la vida cotidiana locales, para analizarlas conceptualmente y enriquecer nuestra comprensión de lo humano. En todo caso, los "productos" finales de esta metodología serán dos y no uno solo: por un lado, la producción científica, que sirve a los fines del/la investigador/a; por otro lado, herramientas concretas para enfrentar incompletitudes locales.

\section{Conclusión}

La intención de este artículo consistió en repensar los alcances pero también algunos desafíos metodológicos del programa de la IAP, mediante un ejercicio de extrañamiento etnográfico sobre procesos de IAP con comunidades indígenas de la provincia de Jujuy en los que yo mismo he participado. Mediante este ejercicio, 
he podido visualizar la existencia de dos roles de mediación social que ocurren en situaciones de IAP o de técnicas participativas en general: la tarea de los/as animadores pedagógicos, y la de los/as agentes de estimulación, esta última ejercida por sujetos empapados en las tramas sociales locales, tales como docentes, dirigentes comunitarios, agentes sanitarios, o técnicos/as de terreno. La tarea del/la agente de estimulación permite el acceso a un conocimiento detallado y capilar del campo social, por medio de dispositivos metatallerísticos y epitallerísticos que trascienden los quehaceres específicos de la instancia del encuentro colectivo (el taller, la asamblea, etc.).

A partir de esta reflexión, y recuperando la propuesta metodológica de la IAP pero repensándola a la luz de una aproximación etnográfica a la categoría de participación, propuse otra forma alternativa posible de movimiento epistemológico de la IAP, no necesariamente en términos de producción colectiva de un conocimiento de coautoría, sino más bien en términos de una reciprocidad explícita entre agentes académicos y agentes locales o sujetos del campo social de análisis. De esta manera, invito a pensar la aproximación del/la investigador/a social al campo social de análisis en tanto agente de estimulación, empapado/a en las tramas sociales locales, para participar activamente en la identificación, verbalización y enfrentamiento de las incompletitudes de la vida cotidiana local. Reformulo así el ciclo dialéctico acción-reflexiónacción propuesto por la IAP, desde una perspectiva etnográfica, en tanto observación-participaciónobjetivación, en los siguientes movimientos metodológicos: observar las incompletitudes-participar en su enfrentamiento-objetivar las configuraciones del sentido práctico y la vida cotidiana locales.

\section{ReFERENCiAS}

Achilli, E. L. (2005). Investigar en antropologia social. Los desafios de transmitir un oficio. Rosario: Laborde Libros.

Arribas Lozano, A. (2015). Antropología colaborativa y movimientos sociales: construyendo ensamblajes virtuosos entre sujetos en proceso. Ankulegi. Revista de Antropología Social, 19. Descargado de: https://aldizkaria.ankule gi.org/index.php/ankulegi/article/view/77 >

Barreteau O., Bousquet F., Étienne, M., Souchère, V. \& d'Aquino, P. (2010). La modélisation d'accompagnement: une démarche participative en appui au développement durable. Collection Update Sciences \& Technologies, ed. Quae. Recuperado de: https://www.researchgate.net/publication/50403465_La_modelisation_d'accompagnement_ une_methode_de_recherche_participative_et_adaptative/download

Batallán, G. y García, J. F. (1992). Antropología y participación. Contribución al debate metodológico. Publicar en Antropologia y Ciencias Sociales, I(1).

Boivin, M., Rosato, A., Arribas, V. ([1989] 2007). La observación participante. En Constructores de Otredad. Una introducción a la Antropología Social y Cultural (pp. 187-204). Buenos Aires, Editorial Antropofagia.

Boletín del IPAF s/f. La Investigación Participativa en el IPAF. En Boletín del Instituto de Investigación y Desarrollo Tecnológico para la Pequeña Agricultura Familiar (IPAF) -Región NOA- del INTA. Disponible en http://a gro.unc.edu.ar/ extrural/IPAF.pdf

Bourdieu, P. (2007). El sentido práctico. Buenos Aires: Siglo XXI Editores.

Carenzo, S. y Fernández Álvarez, M. I. (2014). De la investigación-acción a la etnografía colaborativa. Aportes para (re)pensar el vínculo con organizaciones sociales desde ámbitos universitarios. En M. Gómez Solorzano y C. Pacheco Reyes (eds.), Trabajo informal, economía solidaria y autogestión. Precariedad laboral y resistencia en la globalización (pp. 145-149). Buenos Aires: Ediciones Continente.

Citro, S. (1999). La multiplicidad de la práctica etnográfica: reflexiones en torno a una experiencia de campo en comunidades tobas. Cuadernos del Instituto Nacional de Antropología y Pensamiento Latinoamericano, 18, 91-107

Cladera, J. L. (2010). Cuando propiciar es jugar. Una reflexión sobre los rituales andinos en las comunidades rurales de Salta y Jujuy. En Enrique N. Cruz (Comp.), Carnavales, Fiestas y Ferias en el mundo andino de la Argentina (pp. 110-136). Purmamarka Ediciones y CEIC (FHyCS - UNJu): S.S. Jujuy. 
Cowan Ros, C. y Nussbaumer, B. (2011). Trayectoria conceptual de la mediación social: expedicionarios, patrones, políticos y profesionales técnicos en la interconexión y producción de mundos y significados. En B. Nussbaumer y C. Cowan Ros (editores). Mediadores sociales en la producción de prácticas y sentidos de la política pública (pp. 17-68). Buenos Aires: Ciccus.

Equipo de Sistematización de la DATyC. (2013). Introducción. En Argentina. Experiencias de desarrollo rural. Sistematización y revalorización de saberes. Buenos Aires: Subsecretaría de Agricultura Familiar, Imprenta del MAGyP de La Nación.

Fals Borda, O. ([1979] 2015). Cómo investigar la realidad para transformarla. En V. M Moncayo (antología y presentación), Orlando Fals Borda. Una sociología sentipensante para América Latina. México, D. F. y Buenos Aires: Siglo XXI Editores y CLACSO.

Fals Borda, O. ([1998] 2015). Experiencias teórico-prácticas. En V. M. Moncayo (antología y presentación), Orlando Fals Borda. Una sociología sentipensante para América Latina. México, D. F. y Buenos Aires: Siglo XXI Editores y CLACSO.

Fernández Álvarez, M. I. y Carenzo, S. (2012). "Ellos son los compañeros del CONICET": el vínculo con organizaciones sociales como desafío etnográfico. Publicar en Antropologia y Ciencias Sociales, 10(12). Disponible en http://ppct.caicyt.gov.ar/index.php/publicar/article/viewFile/1562/3003

Fernández Álvarez, M. I. y Carenzo, S. (2014). Del “otro" como sujeto de investigación al "otro" como productor de conocimiento: (re)pensando la práctica de investigación etnográfica con organizaciones sociales. Encuentro de Saberes, 5. Buenos Aires: Ediciones FFyL, UBA. Descargado de https://ri.conicet.gov.ar/handle/11336/45930

Food and Agriculture Organization (2004). Guia Metodológica de Sistematización. Honduras: Programa Especial para la Seguridad Alimentaria.

Freire, P. ([1973] 2001). ¿Extensión o comunicación? La concientización en el medio rural. Buenos Aires: Siglo XXI Editores.

Freire, P. (2006). Pedagogía del oprimido. Buenos Aires: Siglo XXI Editores.

Guber, R. (2004). El salvaje metropolitano. Reconstrucción del conocimiento social en el trabajo de campo. Buenos Aires: Paidós.

Isla, A. y Colmegna, P. (2005). Introducción: política y cultura en las intervenciones de desarrollo. En P. Isla y P. Colmegna (comps.), Politica y poder en los procesos de desarrollo. Buenos Aires: Editorial de las Ciencias.

Jara, O. (s/f). El desafío politico de aprender de nuestras prácticas. Costa Rica: Centro de Estudios y Publicaciones Alforja. Leyva Solano, X. (2010). ¿Academia versus Activismo? Repensarnos desde y para la práctica-teórico-política. En Leyva Solano, et al., Conocimientos y prácticas políticas: reflexiones desde nuestras prácticas de conocimiento situado (pp. $\mathrm{s} / \mathrm{n}$ ). Chiapas México DF, Lima y Ciudad de Guatemala: CIESAS, PDTG-USM, UNICACH. Recuperado de: https://es.scribd.com/document/208382181/Xochitl-Leyva-Academia-contra-activismo

Montero, M. (2006). Hacer para transformar. El método en la psicología comunitaria. Buenos Aires: Editorial Paidós.

Nun, J. (1989). Elementos para una teoría de la democracia: Gramsci y el sentido común. En La rebelión del coro. Estudios sobre la racionalidad politica y el sentido común. Buenos Aires: Ediciones Nueva Visión.

Quirós, J. (2011).El porqué de los que van. Peronistas y piqueteros en el Gran Buenos Aires (una antropología de la politica vivida). Buenos Aires: Editorial Antropofagia.

Rappaport, J. (2007). Más allá de la escritura: la epistemología de la etnografía en colaboración. Revista Colombiana de Antropologia, 43, 197-229. Descargado de http://www.redalyc.org/pdf/1050/105015277007.pdf

Regazzoni, J., De Pascuale, J., Martinez, J. y Virgili, R. (2011). La Investigación Acción Participativa como herramienta para el diseño de máquinas destinadas a la agricultura familiar. Informe de actividades. AER Perico, INTA. Disponible en https://inta.gob.ar/sites/default/files/script-tmp-trabajo_iap_como_herramienta_para_el_dise o.pdf

Rigal, L. (2008). La Investigación Acción Participativa. Buenos Aires: Documento de trabajo de CIPES. 
Rigal, L. (2011). Gramsci, Freire y la educación popular: a propósito de los nuevos movimientos sociales. En F. Hillert, D. H., Suárez, L. Rigal, L., H. Ouviña, M. Gadotti, Gramsci y la educación: pedagogía de la praxis y políticas culturales en América Latina. Buenos Aires: Novedades Educativas.

Ruiz L., Arroyo, S., Jiménez, S., Chuchuy, M., Velázquez, I., Alpiri,... Cladera, J. L. (2013). Mujeres albañiles: el proyecto Yaguata Kavi de la Comunidad Guaraní APG El Bananal (Yuto, Jujuy). En Argentina. Experiencias de desarrollo rural. Sistematización y revalorización de saberes. Buenos Aires: Subsecretaría de Agricultura Familiar, Imprenta del MAGyP de La Nación.

Shugurensky, D. (1989). Introducción al mundo de la promoción social. Michoacán: CREFAL, UNESCO-OREALC.

Sirvent, T. (2004). La investigación social y el compromiso del investigador: contradicciones y desafíos del presente momento histórico en Argentina. Revista del IICE, 22.

Sirvent, T.y Rigal, L. (2014). La investigación acción participativa como un modo de hacer ciencia de lo social. Decisio, (38). Recuperado de https://cdn.crefal.org/CREFAL/revistas-decisio/decisio38_saber2.pdf

Slocum, N., Elliott, J., Heesterbeek, S. y Lukensmeyer, C. J. (2006). Méthodes participatives. Un guide pour l'utilisateur. Bruselas: Fondation Roi Baudouin. Recuperado de: http://www.kbs-frb.be/uploadedFiles/KBSFR B/Files/FR/PUB_1600_MethodesParticipatives.pdf

Sorroche, S. y O'Hare, P. (en prensa). Countering Dispossession through Cooperativisation? Waste-picker Ethnography, Activism, and the State in Buenos Aires and Montevideo. D. Kapoor y S. Jordan (editores), Research and political Engagement in Contexts of Dispossession: Indigenous, Peasant and Urban Poor Activisms in the Americas and Asia. Londres y Nueva York: Zed Books.

Vargas, L. y Bustillos, G. (1984). Técnicas participativas para la educación popular. San José de Costa Rica: Centro de Estudios y Publicaciones ALFORJA.

Vargas, L. y Bustillos, G. (1997). Técnicas participativas para la educación popular. Tomo II. Buenos Aires: CEDEPO y Lumen - Humanitas

Verger Planells, A. (s/f). Sistematización de experiencias en América Latina. Una propuesta para el análisisy la recreación de la acción colectiva desde los movimientos sociales. Universidad Autónoma de Barcelona. Disponible en http:// centroderecursos.alboan.org/ebooks/0000/0774/6_VER_SIS.pdf

Viola Recasens, A. (2000). Introducción: la crisis del desarrollismo y el surgimiento de la antropología del desarrollo. En Antropología del Desarrollo. Teorías y estudios etnográficos en América Latina. Barcelona: Paidós.

Villavicencio, R. (2009). Manual Autoinstructivo: Aprendiendo a sistematizar. Las experiencias como fuentes de conocimiento. Seminario Escuela para el Desarrollo, Agencia Alemana de Cooperación Internacional (GTZ), Programa Desarrollo Rural Sosten

\section{Notas}

1 Y también con comunidades indígenas de territorios aledaños de la provincia de Salta (dpto. Iruya).

2 Programa Social Agropecuario. Constituyó el germen de lo que luego se convertiría en Secretaría de Agricultura Familiar (o Subsecretaría, dependiendo del período político de referencia).

\section{BY-NC-SA}

